\title{
Reducing Noise in the MSU Daily Lower-Tropospheric Global Temperature Dataset
}

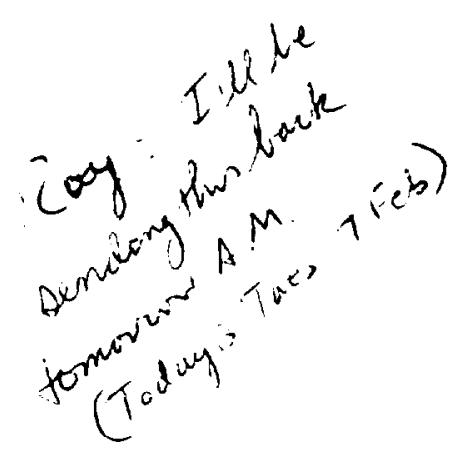

\author{
JOHN R. CHRISTY

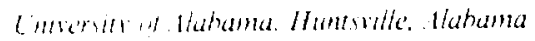 \\ ROY W. SPENCER \\ $\therefore$ St Marshall Space Fligh Cemer. Humsuille. Alabama \\ RICHARD T. MC NIDER \\ Cniversuly of tlahama. Muntswlle. Hahama \\ (Manuscript received 2 May 1994, in final form 25 August 1994) \\ ABSTRACT
}

$1 N-4=-11$

\begin{abstract}
The daily global-mean values of the lower-iropospheric temperature determined from microwave emissions measured by satellites are examined in terms of their signal. noise. and signal-to-noise ratio. Daily and 30 -day average noise estimates are reduced by almost $50 \%$ and $35 \%$, respectively. by analyzing and adjusting (if necessary) for ertors due to 1 ) missing data. 2i residual harmonics of the annual cycle unique to particular satellites. 3) lack of filtering. and 4 ) spurious trends. After adjustments. the decadal trend of the lower-tropospheric global temperature from January 1979 through February 1994 becomes $-0.058^{\circ} \mathrm{C}$. or about $0.03^{\circ} \mathrm{C}$ per decade cooler than previously calculated.
\end{abstract}

\section{Introduction}

Microwave Sounding Units (MSUs) onboard NOAA polar orbiting satellites have provided temperatures of deep layers of the global atmosphere since 1979 [Spencer and Christy (1992a.b. 1993) (hereafter SC92a. SC92b. and SC93)]. These studies have demonstrated the high quality of the MSU data and their value in addressing global climate issues [see also Hurrell and Trenberth (1992). Basist et al. (1995)]. In general. such studies have used MSU data averaged into monthly timescales. In this paper, we shall examine the signal and noise characteristics of the MSU channel 2 retrieval of the lower-tropospheric temperature. known as MSU 2R, for dail! global-mean values. Our goal is to provide a dataset of daily global temperatures for which errors. or noise. have been reduced as much as possible while preserving the magnitude of the signal variability.

The time series of daily zonal-mean temperatures through February 1994 has utilized measurements from instruments on seven different satellites (TIROSV. $10.1 .1-6,-7,-9,-10,-11$, and -12$)$. How these data were generated and merged into a single time series will be explained in the following two sections. To assess changes made to the original data. we shall begin

Cimreynending authur uddres. Mr. John R. Christy. Earth Sistem Science Laboratory. Universitv of Alabama. Huntsville. AL 35899. our analysis from the point where no quality control has been applied. We shall examine the impacts of missing data, unique satellite characteristics. filtering. and spurious trends on the global-mean values and determine how to enhance the quality of the time series. Users who have performed research on current editions of the MSU $2 \mathrm{R}$ data should realize that some qualits control was applied to those data and that the tinal results of this study do not alter the earlier monthly versions in a significant manner.

\section{MSU temperatures}

Temperatures are produced from the raw MSU data in the following way. The radiance counts observed by the MSUs are converted into brightness temperatures by an interpolation scheme, which is anchored on the warm side by an onboard hot target (measured by a set of redundant platinum resistance thermometers) and on the cold side by the temperature of cold space $(2.7 \mathrm{~K})$. On each scan, the sensor obtains radiance counts for these two targets of known temperature as well as eleven footprints over the earth. The counts observed in the earth views are converted to temperatures using the interpolation scheme derived from the cold and hot targets (Spencer et al. 1990).

As described in SC92b, a limb-retrieval technique is applied to the scan-line data, which results in a vertical temperature profile of the lower troposphere. For this study, the daily data are binned into $2.5^{\circ}$ latitude 

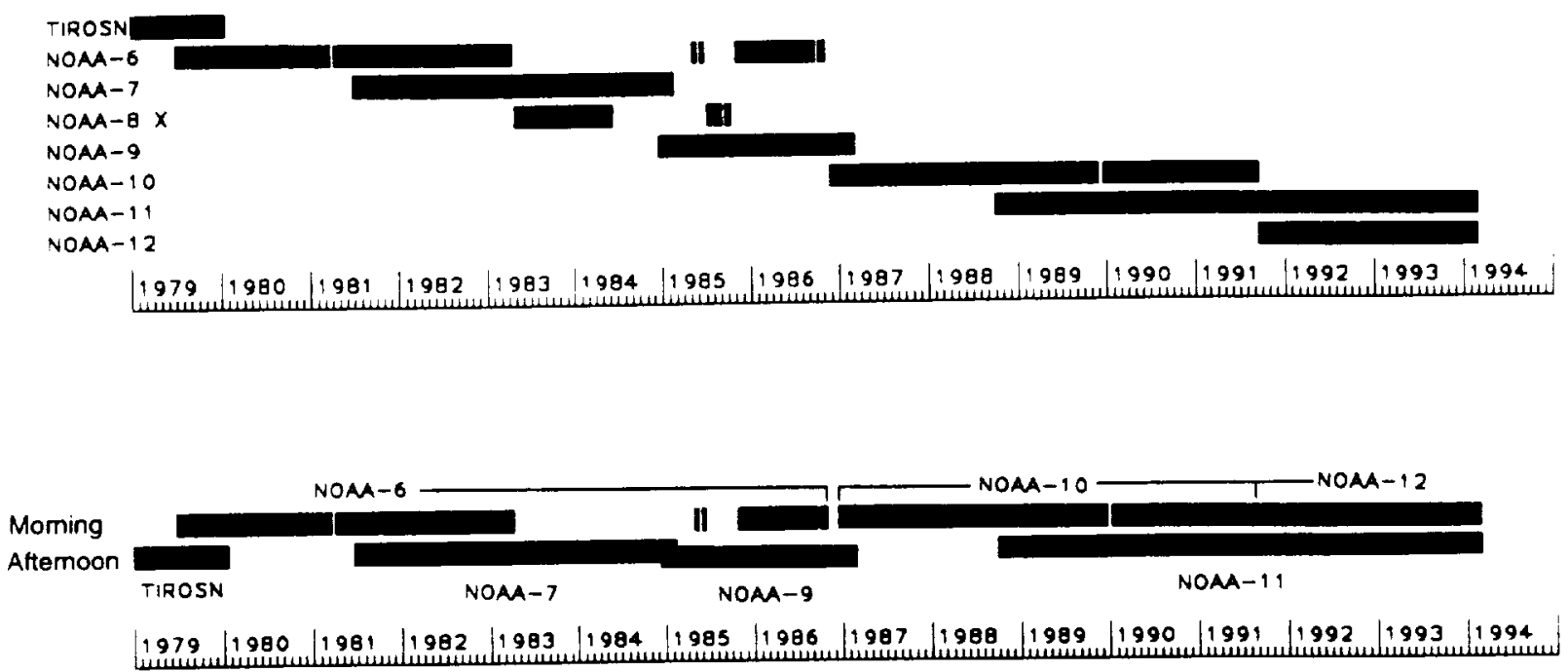

FIG. 1. (Top) Data availability from the operational NOAA polar orbiters. which carried Microwave Sounding Units. (Bottom) As above but categorized by crossing time: morning or afternoon.

bands. which are then averaged into zonal-mean temperatures. Global-mean values are calculated from these zonal quantities. Comparisons with individual radiosonde locations indicate the MSU 2R monthly temperature anomalies correlate most highly with the 1000-500- or 1000-400-hPa thickness anomalies (SC92b). The use of daily zonal means provides a robust dataset from which to calculate global means relative to daily gridded data. which have coarser resolution and much greater noise.

The time period examined here is I January 197928 February 1994. For this $15+$ year period. over $97 \%$ of the possible days had at least $85 \%$ of the possible observations recorded by at least one satellite. Most of the days below the $85^{\circ} \%$ daily data rate occurred between 1979-81 (inclusive).

\section{Merging method}

A brief description of the method by which the data from seven satellites were merged is contained in the Appendix of SC92a. In the following discussion, we shall provide background information and an update of that method.

There are basically two sun-synchronous satellite orbits. which meet the operational requirements of the National Weather Service. One is the "terminator" orbit in which the satellite monitors the earth at local time ( $L T$ ) near sunrise and sunset. crossing the equator at about 0730 LT (descending or southbound) and 1930 LT (ascending or northbound). A platform in this orbit is commonly called the "morning" satellite. The second or "afternoon" orbit crosses the equator at about $1330 \mathrm{LT}$ (ascending ! and $0130 \mathrm{LT}$ (descending). The crossing times for the morning satellites have been very stable through the years. while those of the afternoon orbiters have tended to degrade. ascending later in the day as length of service increases. In particular, the most recent platform. NO.44-II, has drifted by 3 hours. creating potentially serious consequences as described later.

Since 1979, there have been three reliable MSU' on the morning satellites: NO.4A-6, -10, and -12 . The NOAA-8 data proved to be too noisy for necessiry measurements, so the older morning satellite already in orbit (NOAA-6) was returned to operational activities until NOA.4-10 was launched. None of the 10.1 .1 8 measurements were used in any of our previous studies nor will they be used here. The four afternoon satellites are TIROS-N. NOA.A-7, -9, and -11 .

The National Weather Service has generally launched a replacement satellite every 2 years or so. alternating between the morning and afternoon orbits. As such. the operational instruments onboard are nominally anticipated to provide information over a 4-vear period. This schedule has been interrupted due to such circumstances as NO.A.4-8's instability, a malfunctioning oscillator in the MSU on NO.4.4-9. and loss of an entire satellite, as in the August 1993 attempted replacement of NO.A.A- /I with NO.4.4-13. As a result. some instruments have been used for at least 6 years $(N O A 4-6$ and $-l l)$. Figure 1 indicates the sequence of launches and the data used in construction of the $15+$ year MSU time series (NOA.A-8 included for display purposes). Note that there are some periods prior to 1988 in which only one MSU was providing data for the time series.

The instruments are also arranged by crossing time category in Fig. 1. revealing the opportunities where simultaneous comparisons between MSUs on different satellites can be performed. It is in these overlapping periods where a robust measurement of precision can 
be made because the instruments on different satellites are completely independent.

To combine the entire record into a single time series of daily anomalies, two forms of biases were calculated and then removed from each MSU's data in sequence. The first bias is due to differences in the mean annual cycle as observed by the morning orbiters versus that viewed by the afternoon orbiters because they each sample the local diurnal cycle at different times. The greatest morning/afternoon satellite differences occur where surface emissions have large diurnal variations. since the total emission observed by the MSUs includes some emission from the surface. This is especially true over elevated terrain where the fraction of st: ace emission becomes larger, as viewed from the sateilite. because in such locations there is less atmospheric mass to produce emissions. Factors. which determine the amount of surface emission, are surface skin temperature and to a lesser extent. wetness, snow cover, and vegetation. Variations in these parameters. particularly skin temperature. will disproportionately influence the MSU-observed brightness temperature over the course of a 24-hour day.

In general. the ascending node of the afternoon satellite (about 1330 local time) views sun-exposed land and the overlying atmosphere as being warmer than the other passes because of the increase in surface emission (warmer skin temperature) relative to atmospheric emission. This is most apparent over dry regions with considerable sun exposure (Sahara Desert) or over high terrain (Tibetan Plateau) because day-tonight differences in surface skin temperature are much larger than those of the troposphere as a whole. This is a systematic effect and must be treated separately for the afternoon versus morning satellites. [The differences in emission from water surfaces (i.e.. oceans) between day and night is not a factor.] Therefore. to calculate anomalies of atmospheric temperature. we must determine the systematic biases of the afternoon MSUs versus those of the morning MSUs due to diurnal effects such as surface emissions.

We calculate a reference annual cycle based on September 1981-August 1982, a period in which the morning ( NOAA-6) and afternoon ( NOA.4-7) contain adequate data on nearly every day. Daily temperatures for this period are smoothed with a cosine filter (halfpower trequency of 30 days) for each satellite. and the result is defined as the "preliminary" mean annual cycle. Because interannual variability exists. we are required to utilize a period common to both an afternoon and morning satellite, which is at least 12 months long (i.e.. we can not use a different 12-month period for the morning than for the afternoon satellite). Due to serious problems (to be discussed later). $10.4 .1-1 /$ is unavailable to serve as the afternoon satellite and only VO. $1 .-$ contains a period of overlap longer than 1 vear with a morning satellite (Fig. 1 ). Hence. the period detined above is chosen for the preliminary mean an- nual cycle. As noted below, a longer base period will be selected for the final product so that peculiarities of September 1981-August 1982 will not unduly affect the data.

We standardize all of the satellite data by subtracting the morning mean annual cycle from NO.4. $-6 .-10$. and -12 and the afternoon mean annual cycle from TIROS-N, NOA.4-7, -9. and -11 . In this way, we eliminate the diurnal effects peculiar to morning or afternoon orbits from all satellite data. (The reader should keep in mind, however. that what has been removed are annual cycles as observed by only NO.4.4-6 and -7 . These may differ in slight ways from the other instruments had they operated during the 1981-82 period.) At this point, the total time series is a set of anomalies from the mean annual cycle as detined above.

The second bias to be removed arises from the systematic offset of one instrument versus another. Though the daily global anomalies of two simultaneous MSUs may correlate at $0.99+$, each instrument has a relative temperature bias of as much as $1 \mathrm{k}+\mathrm{s}(9 \mathrm{~g})$. SC92b. SC93). We determine these systematl hiases as the average difference between temperature of the MSUs at each latitude during common permat at inservation. The bias is sequentially accumuldicl and removed for each MSU so that all anomalses then are relative to $N O A A-6 / 7$ for September $198:-1.2 .4$ 1982, our preliminary mean annual cycle.

The final step is to select a representative fermut is the base annual cycle and calculate anomalic $r .4$ : . to this. We selected 1982-91 as the base hecius ..... were several missing-day periods prior to 1 w 2.4 .4 length of 10 years seemed appropriate. though $1, \ldots x$ it is arbitrary. We then determined the residuat d.?. . i.di cycle in those 10 years and subtracted this $11, \mathrm{~m}$ ' $: \mathrm{s}$ entire record. The result is $15+$ years of daily anum.dic relative to the 1982-91 period.

\section{Precision during overlap periods}

Because the atmosphere is being observed hr : independent instruments during the overlap perinds. we may determine the signal and noise characteristics of the instruments. In Fig. 2 we show results of the mean and mean difference of the global anomalies of the NOAA-11 and -12 overlap (hereafter $11 / 121$. We shall define noise $(N)$ as the variance of the anomaly difference divided by 2, $N=\left(\sigma_{1 / 2}\right)^{2}$, and the signal $(S)$ as the variance of the anomaly sum divided by 2 . $S=\left(\sigma_{y / 2}\right)^{2}$. The daily $N$ of $11 / 12$ is $0.005565 \mathrm{~K}^{2}$. while the daily $S$ is $0.032184 \mathrm{~K}^{2}$, giving a signal-tonoise ratio $(S / N)$ of only 5.8 . The noise, signal. and $S / N$ for 30 -day averages are $0.001211 \mathrm{~K}^{2}, 0.016512$ $\mathrm{K}^{2}$. and 13.6. respectively. These are the lowest (worst) $S / \mathrm{V}$ values of the six overlaps that are used in merging the dataset into a single time series. Improvements in $S / N$ will be most evident in this example. 


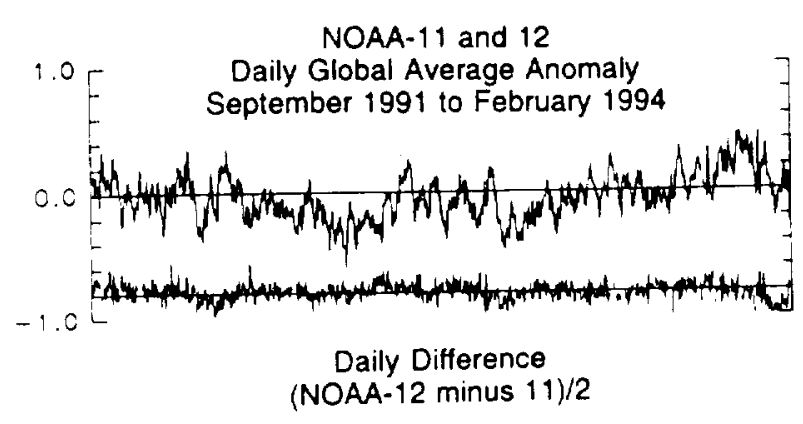

FIG. 2. (Top) The average of the daily global-mean anomalies for 10.1. 11 and -12 (Bottom) The difference of the global-mean daily anomalies (offset by $-0.8^{\circ} \mathrm{C}$ for clarity) divided by 2 . This was the norst case of the six overlap comparisons.

Our goal is to reduce the noise without compromising or smoothing the signal. We note that the MSU datasets previously provided to the scientific community contain some quality control aspects and, in reality, the final result of this present study will not materially alter any conclusions one may have deduced using older versions of the MSU $2 \mathrm{R}$ data. We are showing, here, the results of data analysis for which no quality control was performed. and then we shall introduce measures that will optimize the $S / N$ for both daily and 30-day mean statistics. The effects. which relate to noise reduction are 1 ) variations in the amount of missing data allowed, 2) mean annual cycle differences between $N O .4 .6$ or -7 and the other satellites. 3) median latitudinal filtering, and 4) removal of VO.A.II's spurious warming trend.

\section{a. Impact of data availability}

The information for the statistics presented above for $11 / 12$ were derived from MSU 2R data for which at least 20000 observations per day were available from both satellites. For this section, we use as our "number of observations per day," the total number of footprint observations from which the MSU $2 R$ retrieval is derived. There are about 3000 useful scan lines per day. each of which provides eleven footprint observations. The MSU 2R retrieval scheme utilizes only eight of these observations for the scan-line retrieval, hence. the maximum number of distinct observations on a given day is about 24000 (SC92b). The 20000 value then represents about $85 \%$ of the total number of observations that are possible.

Since one complete orbit provides $7^{\circ} \mathrm{r}$ of a day's data. errors are introduced by subsampling the earth using daily data amounts that represent fewer than 14 orbits. This is especially true where the surface emissions are strong. These areas require systematic sampling each day so as not to introduce biases into the global mean. Even missing only that part of the orbit which views these "hot spots" can lead to greatly increased noise.
We first attempt to reduce $N$ by assessing how missing data impact global-mean temperature values. We have calculated $\sqrt{\bar{N}}=\left(\sigma_{د / 2}\right)$ for 19000,20000 . 21000.22000 . and 23000 per day data rates for daily variations and 30-day average variations (Fig. 3 ). The values plotted are those of a weighted mean of $V I$ for all six overlaps based on the number of days in each overlap period ( recall we have mentioned the statistics of the worst overlap example earlier). The total number of days on which both of the intercompared MSUs reached acceptable data rates were 3009. 2895. 2697. 2133 , and 1436 for 19000 through 23000 . respectively.

The results of the daily comparisons indicate that the noise is reduced as the number of observations required for comparison is increased. as would be expected. The reduction is noticeable but relativels slight with daily $\sqrt{N}$ decreasing from $0.068 \mathrm{~K}$ to $0.0 \mathrm{f}+\mathrm{K}$ for $19000-22000$ data rates. There seems to be no significant difference in $V N$ due to data avaliahilut for 30-day averages, all being about $0.026 K$ ()t . .l! the characteristics we shall examine in this visl: inta availability has the weakest impact on $1 \quad 1 \quad \cdots: m$ result, there is no compelling reason to $1: \ldots, \cdots, \cdots$ ceptable data rate beyond 20000 , whict? currently used in monthly reports (e.g.. '. mate Diagnostics Bulletin).

\section{b. Residual annual clcle differences}

We have indicated that the morning am: instruments observe the earth under differn. conditions, leading to different mean annı.. was assumed that all morning satellites $u_{1} \ldots \ldots$ : identical mean annual variations observe: 6 , and all atternoon satellites those of $\mathrm{NO} / 1$ examination of Fig. 2 reveals a series of . $^{\prime} . \therefore$. . in the difference time series, which happen $1 \cdot \cdot$ locked with the annual period. Because Fig : : : . the last overlap period. in which the MSt $\quad .$. .

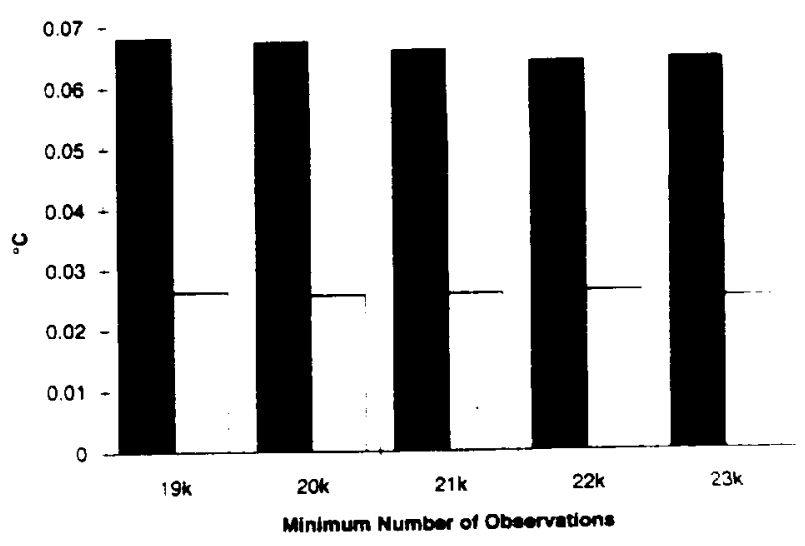

FIG. 3. Solid (open) columns represent $\sqrt{\pi}$ for daily $30-d a\}$ mean I statistics. The left-to-right progression indicates results for minimum daily observation rates of $19 \mathrm{k}, 20 \mathrm{k} .21 \mathrm{k} .22 \mathrm{k}$. and $23 \mathrm{k}$.
Ed:

shor

radie.

be se

$\gamma 0$.

$\gamma$ ? or

ox

mis. 


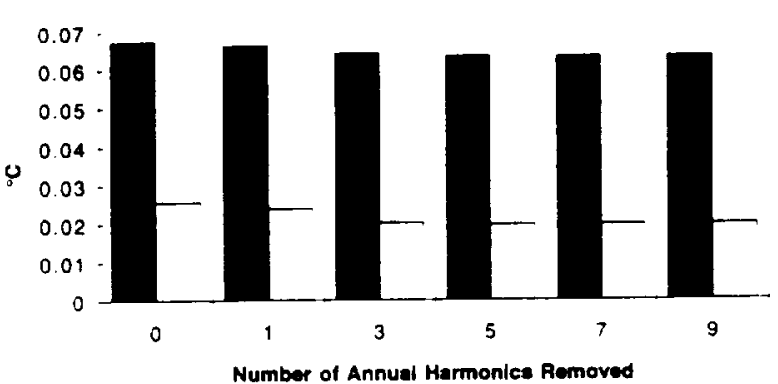

FiG. 4. Solid (open) columns represent $V \bar{T}$ for daily (30-day mean) statistics. The left to right progression indicates results for removal of Fourier components of the annual period up to the wavenumber indicated below the columns.

adjusted with the accumulations of all previous adjustments, any systematic errors should be most visible here. Inspection of all overlap examples indicates that the $11 / 12$ is clearly the only period with such obvious periodicity in the difference time series.

Upon closer study we found that for a given latitude. the NOA. -12 MSU generates warmer than expected temperatures in local winter and colder than expected in local summer. In other words. the annual range of temperatures in the NO.A.A-12 MSU was only about $98 \%$ of the other MSUs. This observation might be related to the fact that the dynamic range of the NOAA$12 \mathrm{MSU}$ channel 2 was reduced due to much reduced gain (Mo 1994).

To account for this effect. that is. adjust the dynamic range of the NOA.A-12 and possibly other MSUs. we decompose. latitude by latitude, the annual cycle of the difference time series into Fourier components for those overlap cases longer than 1 year (NOAA 6/7.6) $9.10 / 11,11 / 12)$. Once these Fourier components of the residual are removed, the time series is reconstructed as before. The results for daily and 30-day means are shown in Fig. 4 for various Fourier-component wavenumbers removed (e.g., " 5 " indicates removal of components 1 to 5 ).

The removal of Fourier components $0,1,1-3,1-5$. $1-7$. and $1-9$, based on the difference time series. produces $S / N$ values for daily anomalies of 8.7, 9.2. 9.8. 9.9. 10.0 and 10.0, respectively. For 30-day averages. these values are $26.2,30.6,43.3 .45 .2,45.1$, and 45.1. This adjustment impacts the 30 -day $S / N$ substantially more than the daily, reducing $V N$ by over $20 \%$ in the case of waves $1-5$. The daily $\sqrt{N}$ is also reduced. but less than $10 \%$ because its fluctuations occur more on a day-to-day basis. There is no significant reduction of $N$ bevond wave $1-5$ removal. Given this magnitude of noise reduction we believe this procedure should be applied ( up to annual harmonic 5 ) to the data supplied to the users. The process of removing Fourier components of the annual period does not affect the overall trend of the time series at the $0.002{ }^{\circ} \mathrm{C}$ per decade level and has its primary impact on NOA.t-IS.

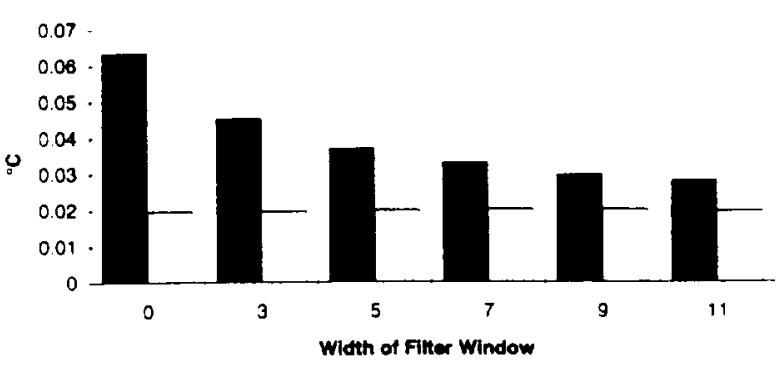

FIG. 5. Solid (open) columns represent $\sqrt{N}$ for daily (30-day mean) statistics due to median filtering with window width $2 n+1$ indicated below.

\section{c. Median filtering}

The northern midlatitudes tend to have a large influence on the global-mean anomaly because of the large temperature variations that are observed over the continental regions. When an MSU undersamples this area relative to other areas, there is a potential for dramatically increased noise in the difference between two observing satellites. The spikiness of the time series in Fig. 2 is strongly related to this. Rather than averaging each zonal anomaly across a few days to reduce the noise, we shall use the technique of median filtering in which the outliers or spikes are essentially eliminated from the dataset.

For a given latitude and given day, the median is determined from the current value ranked with $n$ days previous and following. The median window is then $2 n+1$ days in length. Data will be set to missing if the $2 n+1$ window finds less than $n+1$ days of data. In Fig. 5 we show the result of noise reduction to the daily global-mean anomaly for no median filtering through an 11-day window $(n=5)$ once the annual harmonics have been removed as described above. We notice a substantial reduction in daily $\sqrt{N}$ for $n=1$ and 2 , with lesser reductions thereafter. However, reducing $I$ is only one component of the goal, the other being a preservation of the signal magnitude $S$. For the same categories of median filtering, in Fig. 6 we display the values of $V \bar{S}$ where reductions are also apparent in the daily variance. A portion of the reduction in both $N$ and $S$ is due to the removal of the errors in the times

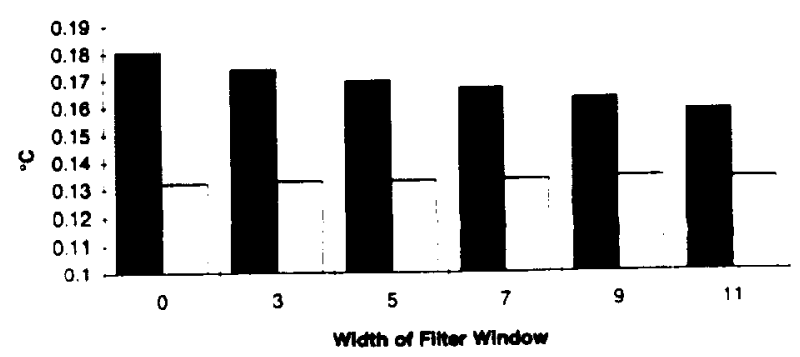

FIG. 6. As above for $V S$. 


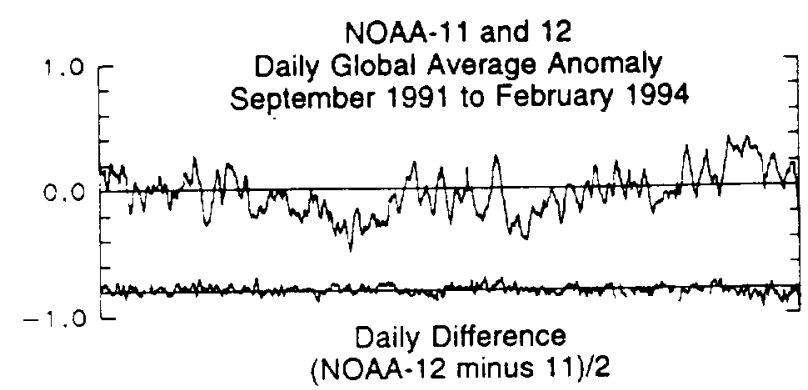

FIG. 7. As in Fig. 2 for the NOA.4-1/ and NO.4.4-12 overlap comparison after removal of five Fourier components of the annual period and inclusion of 5-point median filtering. Note the trend in the difference time series.

series. However, the natural smoothing characteristic of median filtering is also a factor.

We believe it is desirable to include median filtering of the latitudinal anomalies because of the obvious reduction of $N$. The selection of the width of the window is somewhat arbitrary since the level of signal smoothing one might accept varies according to research requirements. We have chosen $n=2$ for a width of 5 . which reduces $\sqrt{S}$ by only $5.6 \%$ while reducing $\sqrt{N}$ by $33 \%$. The $S / N$ ratios of daily global anomalies for the median filter windows shown in Figs. 5 and 6 (width: $2 n+1=0.3,5,7,9$, and 11 ) are 9.9, 18.1, 26.2, 31.8. 38.1 , and 41.1 , respectively. Reductions in $\sqrt{S}$ for the same sequence of values are $0 \%, 3.8 \%, 5.6 \%, 7.0 \%$. $8.4 \%$, and $9.8 \%$. The $S / N$ ratios for 30 -day averages were $45 \pm 1.5$ with no discernable relationship between window width and $S / N$.

A median filter of width 5 would not be expected to replace the center or current value with the calculated median one time out of five ( $20 \%$ ) for a random distribution. In other words, the time-centered value would be the actual median $20 \%$ of the time. For processes. which have discernable variations on time spans longer than 5 days. the percentage of nonreplacement would be greater as the current value would have a higher probability of being the actual median value if there were a trend over the 5-day period.

Analysis of the 5-day median replacement rate of the latitudinal anomalies indicates that about $35^{\circ} \circ$ are not replaced at high latitudes, with that percentage decreasing to slightly more than $20 \%$ at the equator. This implies that tropical variations of zonal-mean temperature are so slow as to represent essentially random distributions on 5-day intervals. Concerning the almost $80 \%$ that are replaced in The Tropics. the difference between the current and median (i.e., replacement) value produces a $\sigma$ of about $0.2^{\circ} \mathrm{C}$. In higher latitudes, the $65^{\circ} c$ current and median values differ with a $\sigma$ of about $0.4^{\circ} \mathrm{C}$. The effect of the latitudinal median filter is rather slight on the global mean as compensation between latitude bands occurs so that the $V \bar{S}$ is reduced by only $5.6 \%$.

The reduction of $\sqrt{N}$ from a combination of har- monic removal and median filtering is over $45 \%$ for daily observations and over $20 \%$ for the 30 -day averages. Figure 7 shows the same overlap of $11 / 12$ as shown in Fig. 2 after these two procedures are applied. For this particular overlap example. the $S / N$ for daily and 30-day averages are now 18.4 and 36.8 , respectively, up from 5.8 and 13.6. Using the various window widths, we calculated the trend of the entire time series and found variations of the trend are less than $0.002^{\circ} \mathrm{C}$ per decade from that of the selected $(2 n+1=5)$ filter.

\section{d. The issue of trends in the differences}

What is now apparent in the $11 / 12$ overlap time series of differences ( Fig. 7) is the disturbing presence of an obvious trend. In actuality, the trend began in 1990 when the temperatures of the $10 / 11$ overlap began to diverge. During the first 2 years of the $10 / 11$ overlap, there was no trend in the difference time series greater than $0.01^{\circ} \mathrm{C}$ per year (Christy and Goodridge 1994). Since 1990, NOAA-11 observes a global temperature trend, which is $0.03^{\circ}-0.04^{\circ} \mathrm{C}$ per year warmer than that of post-1989 NOAA-10 and all of NOA.4-I?

Because NO.AA- $I I$ is in disagreement with two other satellites, our first assumption is that NOAA-II is the source of the error. This suspicion is substantiated by NOAA-1 I's orbital drift from the initial equatorial crossing time of $0130 / 1330$ to $0430 / 1630$ as of February 1994 (Fig. 8). The question now becomes how does the global-tropospheric temperature relate to the changing time of observation (i.e.. diurnal cycle). The troposphere is generally warmer at 1630 than at 1330 but is this warming entirely offset by the cooling that would be expected in the nighttime pass as it drifted from 0130 to 0430 ?

To provide further insight into this issue we calculated the trend latitude-by-latitude for the difference between NOAA-1I and the two morning orbiters. NOAA-1O and -12 (Fig. 9). We see that the subtropical

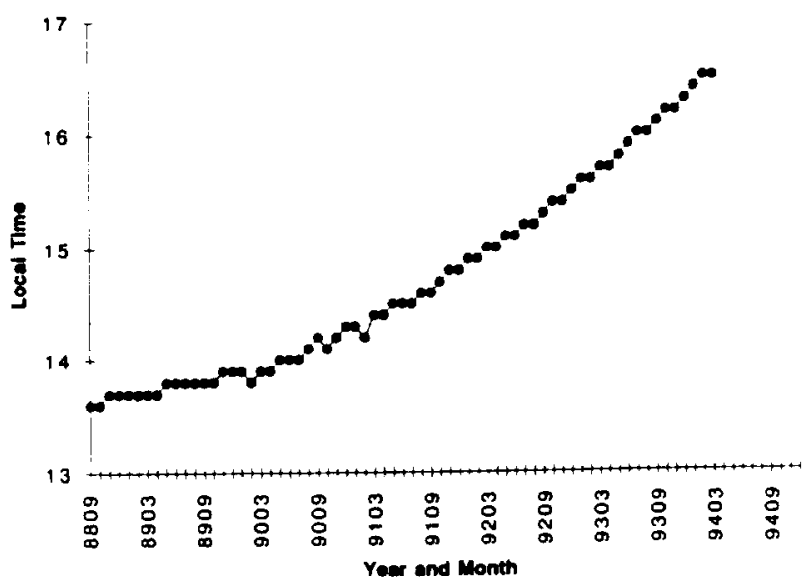

FIG. 8. Ascending pass crossing time at the equator for $1.0 .4 .4 .1 /$ from September 1988 to February 1994 


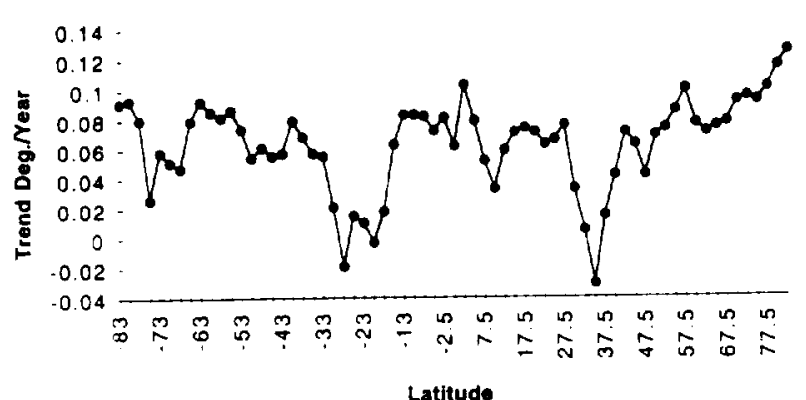

FIG. 9. Relative trend $\left({ }^{\circ} \mathrm{C}\right.$ per year) between NOA.4- $/ I$ versus YO.t.1.10 and NO.t.A.12 determined from 1 January 1990 to 28 Februan 1994 as a function of latitude.

latitudes show slight positive to negative trends for the relative difference. while at all other latitudes the sign is positive. The notion that the MSU on NOAA-II is contributing a spurious warming trend related to its orbit time decay seems confirmed by this figure. In those regions with strong convection, in which surface heat is transferred to the free atmosphere throughout the afternoon, the warming measured from 1330 to 1630 would be greater than cooling during the stable period from 0130 to 0430 . The Northern Hemisphere. with its greater proportion of land surface relative to the Southern Hemisphere, seems to have a greater propensity for vertical mixing and, therefore, generally supports more positive trends during the $0130 / 1330-$ $0430 / 1630 \mathrm{drift}$. Even over those oceanic regions. which are subject to frequent precipitating systems (i.e., tropics and midlatitudes), it has been shown that the tropospheric temperature peaks at about 1600 local time ( Randall et al. 1991).

However, in the subtropical latitudes there is considerable subsidence and, thus, decoupling between the boundary layer (most often a marine boundary layer in the Southern Hemisphere) and the free atmosphere (Trenberth et al. 1992). The diurnal cycle in the troposphere would more closely follow the solar cycle. peaking in the early afternoon and cooling until dawn. Tropospheric temperatures at 0430 would certainly be cooler than those at 0130 , while in the afternoon, the 1630 observation would most likely be equal to or cooler than the 1330 observation.

The latitude bands in the $32.5^{\circ}-40^{\circ} \mathrm{N}$ region have a peculiar character in that they are somewhat affected by the typical subtropical atmospheric processes and also by pronounced surface emissivity effects. The highlands from Iran eastward to China penetrate the MSU weighting function. "shining through" the atmosphere to some extent. The diumal cycle of the MSU $2 \mathrm{R}$ in these areas is very closely tied to the surtace skin temperature, so that the MSU brightness temperature reaches its maximum earlier (near solar noon) than does the physical temperature of the air above. By viewing at 1630 rather than 1330 . NOA.4-11 observes a cooler brightness temperature due to the cooler skin temperature even though the atmosphere there may be warmer. The removal of a negative trend in those bands is, therefore. necessary.

The effect of the satellite orbit decay would be greater during summer. when convection (dry and moist) is more vigorous, than during winter. As such. one would expect an annual cycle in the time-drift effect on the difference time series. This is not seen in our data at this point because such oscillations. which are anchored to the annual period. have already been removed ( section $4 \mathrm{~b}$ ). In fact, that these harmonics were present to begin with seems related to this orbit-drift problem as well as instrument differences.

The trends of Fig. 9 are removed from the NO.A.111 data, beginning with 1 January 1990 . This process further reduces the noise of the last two overlap pairs and. thus, the overall noise levels. The final $\sqrt{N}$ and $S / N$ for all overlapping comparisons are $0.0348 \mathrm{~K}$ ( $0.0167 \mathrm{~K}$ ) and 29.5 (63.4) for daily ( 30 day averages) respectively. The reductions of noise for the procedures described in this paper are summarized in Fig. 10.

Our definition of noise, as the variance of the difference time series divided by two, is not a traditional quantity but did suit our purposes for the visual comparison of signal (the sum divided by two) and noise for intercompared satellite data. The standard error of measurement. as another representation of noise. requires that our $\sqrt{N}$ be multiplied by 1.414 (i.e. $2 / \sqrt{2}$ ). We. therefore, compute the daily (30 day average) value of the standard error of measurement as $0.0492 \mathrm{~K}(0.0236 \mathrm{~K})$.

In Fig. Il we show the $11 / 12$ overlap in which now there is no trend in the difference time series. We note here that the latitudinal trends removed in NO.4.1-11 are linear functions of best-fit. We assumed that the actual warming/cooling rates due to time drift are linear over the 3-hour drift observed in NOA.4-11. As it continues to drift, we expect that the instrument will eventually observe temperatures at their local maximum (globally speaking) of the diurnal cycle and then the temperatures will begin to decline. Since NOA.4$l l$ is degrading at 6 minutes per month, we suspect

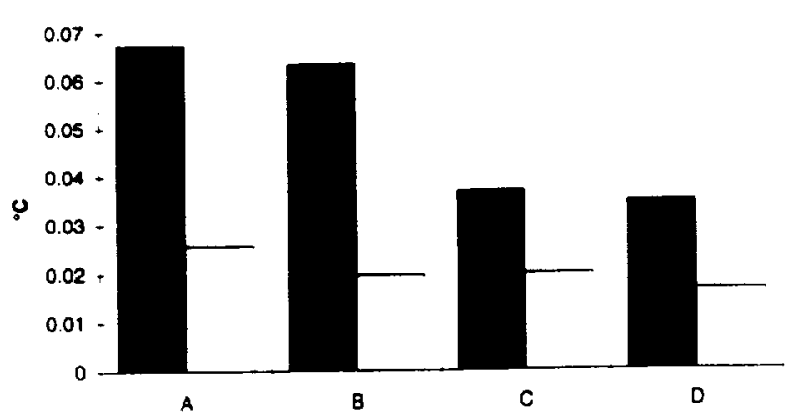

FIG. 10. Solid (open) columns represent $\sqrt{N}$ for daily (30-day averages) for $\mathrm{A}$ : unaltered data for $20 \mathrm{k}$ observations. $\mathrm{B}$ : as in $\mathrm{A}$ with removal of five Fourier components. $C$ : as in B with 5-point median filtering. and $\mathrm{D}$ : as in $\mathrm{C}$ after removal of NO.4.1/1 trend. 


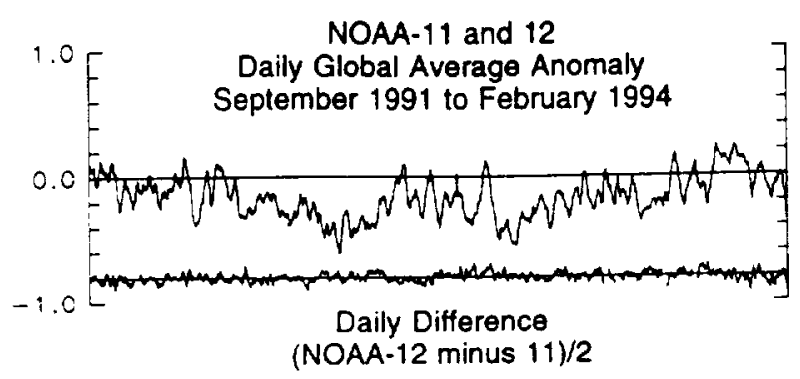

FIG. 11 . As in Fig. 7 for the $10.44-1 /$ and -12 overlap after latitudinal irends of NOAt-II are removed.

that the usefulness of the linear adjustments made here may have already reached their limit.

The orbital drift in the afternoon satellites is not detectible in the temperature data until the third year of orbit, as in the case of NOAA-11. For TIROS-N. there was a sudden drop in the MSU data rate after only 14 months (January 1980), so drifting had no impact. The oscillator on NOAA-9's MSU failed after only 27 months, rendering the instrument inoperative for channel 2. We should note that other instruments remained in operation on NOAA-9 and researchers using those data will certainly have to deal with the problem of drift. The MSU on NOAA-7 was in operation for 3.5 years, and, as such, there may be a slight warming effect late in its term of service (about 1984, Fig. 1) when it was the only MSU in operation. We should note. however, that the subsequent MSU on NOAA-9 was calibrated directly against $N O A A-6$, and so did not require $N O A A-7$ data. The long time series of NOAA6 was rigorously checked by radiosonde data from mid1979 through 1986 and found to have no temperature drift over the 7.5 years ( Spencer et al. 1990). Therefore. the MSU time series after NOAA-7 is independent from any temperature drift that might have occurred between the first shutdown of NOAA-6 (April 1983) and its restart in April 1985.

\section{Conclusions}

Careful examination of the daily global-mean anomalies of the lower-tropospheric temperature through the comparison of simultaneously operating satellites has allowed a direct determination of signal and noise of the data. From this, we have determined which characteristics are important in noise reduction. In the case of missing data, there was little impact found between the 19000 and 22000 per day data rates. so the value of 20000 was retained.

There were significant impacts due to residual annual cycle differences between the various instruments and the lack of latitudinal/temporal filtering. By removing the residual annual cycle to 5 Fourier components and applying a median filter of width 5 days for each latitude, the daily and monthly noise were reduced hy over $40 \%$ and $25 \%$, respectively, while leaving the signal with over $90 \%$ of its original variance.

The time drift of NOAA-II was shown to induce. since 1990, a spurious warming trend in the tume series as it observed the atmosphere at warmer |xial umes (globally averaged) during its degradation. Once $(. .1$. culated and removed, the resultant total tIme series produces a decadal trend for the $15+$ year $11 \mathrm{me}$ x'm of $-0.058^{\circ} \mathrm{C}$ (Fig. 12). This is $0.03^{\circ} \mathrm{C}$ per decude cin wer than calculated under the assumption that $(;)+1 /$ was in a stable orbit. Also in Fig. 12 is the umi x.ris of the differences between previously reporied kluhd temperatures and those using the adjustments $d$ ppils from this current research. Monthly differences arc : irs

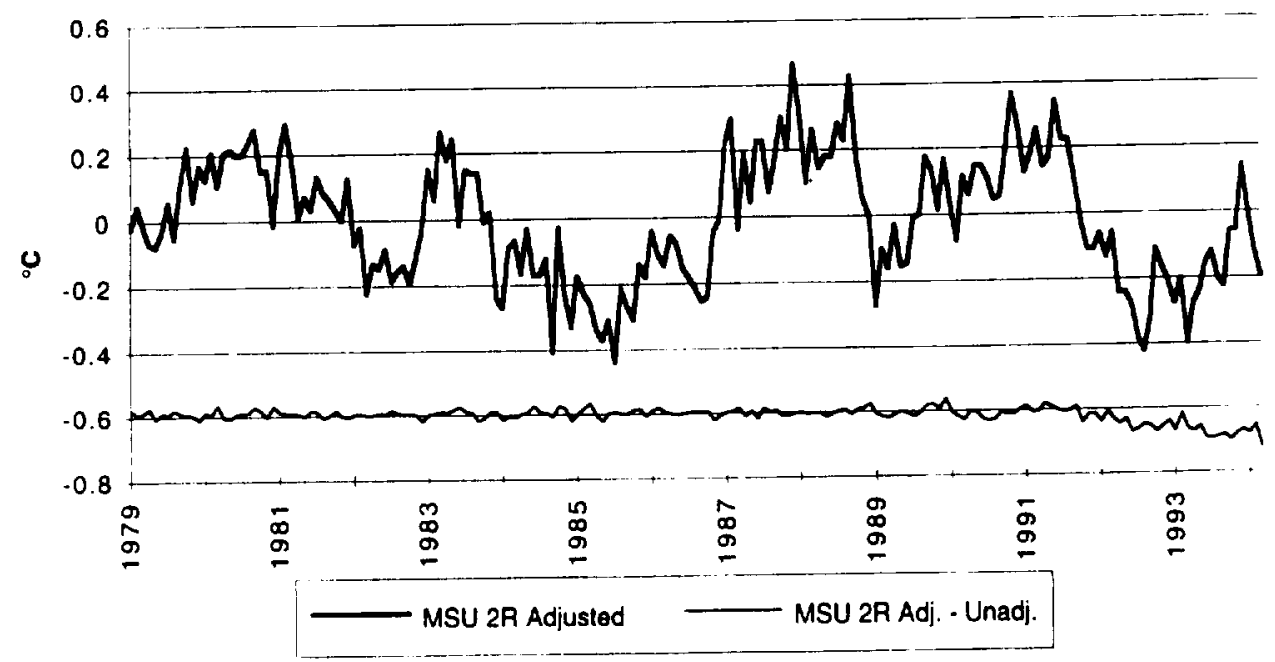

FIG. 12. Time series of monthly global anomalies (thick line) relative to $1982-91$ annual cycle after removal of effects noted in this paper. Difference (thin line, offset by $-0.6^{\circ} \mathrm{C}$ for clarity) relative $10 \mathrm{MSU}$ ?R values reported prior to adjustments given in this paper. The time series here includes March 1994. 
minor (within $0.03^{\circ} \mathrm{C}$ ) until the last 3 years when the impact of the NOAA-1/ drift is apparent.

In a recent report, Christy and McNider (1994) estimated the magnitude of the global MSU $2 R$ temperature trend once the effects of volcanoes and El Niños were removed. The goal was to isolate a possible warming signal due to the enhanced greenhouse effect. Their resultant 15 -year trend was about $+0.09^{\circ} \mathrm{C}$ per decade. Using the MSU 2R data, as adjusted in this study, the volcanic impact was reevaluated because the impact of Pinatubo (June 1991) occurred entirely within the period, which was affected by the NOAA11 orbital drift. The global temperatures since 1991. now cooler than previously thought, imply that Pinatubo had an even stronger impact on the global temperature than estimated in Christy and McNider. However, after reevaluating the volcanic effect on the now-cooler post-1990 global temperatures and then removing that effect we find the "global warming" trend remains at about $+0.09^{\circ} \mathrm{C}$ per decade as of July 1994.

An opportunity is now available to assess the diumal cycle of temperature of the global- (and zonal) free atmosphere due to the inadvertent drift of NOAA-11. With the morning satellites well anchored to the terminator orbit, we can compare global and zonal temperatures in NOAA-II with the morning orbiter data throughout the 3-hour drift as was done in this paper. From this, we may determine average changes between the drifting observations and those of the morning orbiters to assess part of the diurnal cycle. Investigations concerning the diumal cycle, as well as correcting MSU channel 4 (lower stratosphere), will be addressed in future work.
Acknowledgment. This research was supported by the NOAA Climate and Global Change Program and the NASA Mission to Planet Earth Program.

\section{REFERENCES}

Basist. A. N.. C. F. Ropelewski. and N. C. Grody, 1995: A comparison of tropospheric temperatures derived from the NOAA microwave sounding unit and the National Meteorological Center analyses. $J$. Climate, $\mathbf{8}$, (in press).

Christy, J. R.. and J. D. Goodridge. 1994: Precision global temperatures from satellites. Aimos. Env. (in press).

- - and R. T. McNider. 1994: Satellite greenhouse warming. Naiure. 367,325

Hurrell, J. W., and K. E. Trenberth, 1992: An evaluation of monthly mean MSU and ECMWF global atmospheric temperatures for monitoring climate. J. Climate. 5, 1424-1440.

Mo. T. 1994: A study of the microwave sounding unit on the 1. O.t. 12 satellite. Proc. Int. Geoscience and Remote Sinsing Simp. Pasadena. CA. 8-12 August 1994. 1535-1537

NOAA. Climate Diagnostics Bulletin, V. Kousky Ed.. A summary of monthly climate diagnostics obtained from VOA VWS NMC. 5200 Auth Rd. Camp Springs. MD 20748

Randall, D. A., Harshvardhan. D. A. Dazlich. 1991 Durnal :urability of the hydrologic cycle in a general circulustion mindel $J$ Almos. Sci., 48, 40-62.

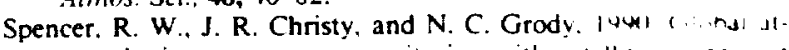
mospheric temperature monitoring with satellite - rous se measurements: Method and results 1979-8t $f$ " "s ? $1111-1128$.

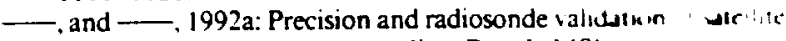
gridpoint temperature anomalies. Part I: MSI, nan Climate, 5, 847-857.

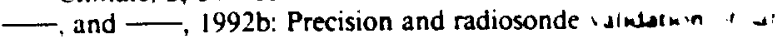
ellite gridpoint temperature anomalies. Part $11+r \ldots w$. retrieval and trends during 1979-90. J. Climari. .......

$\ldots$ and -1993 : Precision lower stratospher.... monitoring with the MSU: Validation and results . . Climate, 6, $1194-1204$.

Trenberth. K. E., J. R. Christy, and J. W. Hurrell, I w4: "I ". . ' global monthly mean surface temperatures. $J($ anc. ' 1423. 